\section{Preliminary results of pharma- comechanical thrombectomy for iliofemoral deep venous thrombosis: A single center experience}

\author{
Mihriban Yalcin, ${ }^{1}$ \\ Eda Godekmerdan Katırcıoglu, ${ }^{1}$ \\ Kamuran Erkoc, ${ }^{2}$ Osman Tiryakioglu ${ }^{2}$ \\ ${ }^{1}$ Ordu State Hospital, Cardiovascular \\ Surgery, Ordu; ${ }^{2}$ Private Medicalpark \\ Hospital, Cardiovascular Surgery, \\ Bursa, Turkey
}

\begin{abstract}
Our aim is to explain follow-up in patients treated with pharmacomechanical thrombectomy (PMT) followed by balloon angioplasty for lower extremity deep venous thrombosis (DVT). A total of 19 patients who underwent PMT for DVT were included in the study. The patients underwent PMT with the Cleaner device after insertion of vena cava filters. The mean age was 45 years (range 20-56). The lesions were on the right side in nine patients and on the left side in 10 patients. Complete thrombus removal was achieved in 14 patients and the remaining five patients underwent balloon angioplasty and stenting to achieve significant patency. In conclusion, our single-center experience with short-term results suggests that PMT with the Cleaner device can be used to successfully manage acute and sub acute DVT.
\end{abstract}

\section{Introduction}

Deep venous thrombosis (DVT) is one of the most common vascular pathology in the world. Hypercoagulability, stasis, and endothelial injury, which are collectively known as Virchow's triad, are risk factors for DVT. Due to the increasing age profile and the increase of medical illness such as cancer, obesity, long haul travel and surgery, it seems that the incidence of venous thromboembolic disease will continue to increase. $^{1}$

Pulmonary embolism, paradoxical embolization and phlegmasia cerulea dolens are major clinical situations that may occur with acute DVT. Recurrent DVT, chronic thromboembolic pulmonary hypertension, and post-thrombotic syndrome (PTS) are the later complications and they may be present despite the use of anticoagulant therapy. ${ }^{2,3}$

PTS develops in $25-50 \%$ of patients with a first episode of proximal lower extremity DVT. ${ }^{2,4}$ The clinic manifestations of PTS are swelling, pain, heaviness, and fatigue of the affected limb. Severe PTS may cause shortdistance venous claudication, stasis dermatitis, hyperpigmentation and subcutaneous fibrosis, and skin ulceration. ${ }^{5}$

It has been reported that the quality of life for patients with severe PTS is as hard as for patients with major chronic illnesses such as angina, cancer and congestive heart failure. ${ }^{6}$

The aims of treatment: i) to prevent thrombus propagation and embolization; ii) to minimize recurrent thrombosis; iii) to relieve obstruction; and iv) to preserve valve function. ${ }^{7}$

The initial therapy for acute lower extremity DVT is subcutaneous lowmolecular weight heparin (LMWH) or intravenous unfractionated heparin (UFH) for at least five days followed by an oral vitamin $\mathrm{K}$ antagonist therapy (international normalized ratio between 2-3) for 3-12 months. Also patients with underlying thrombophilia or those with unprovoked VTE may merit extended duration of anticoagulation if the benefits outweigh the risks.

In the 1960s the use of intravenous thrombolytic treatment and surgical thrombectomy, in the 1980s catheter directed thrombolysis (CDT) and in the 1990s and then percutaneous mechanical thrombectomy were used to remove the thrombus from the deep venous circulation. ${ }^{8}$ Balloon angioplasty and endovascular stent placement are also used to treat residual iliocaval compression.

In the present retrospective study we aimed to evaluate the efficacy and safety of PMT for symptomatic DVT.

\section{Materials and Methods}

We retrospectively reviewed nineteen patients with iliofemoral acute and subacute DVT who were managed with PMT in our hospital between November 2012 and July 2016. Symptom duration was defined as acute (0-14 day), subacute (15-28 days), or chronic ( $>29$ days). A total of 74 patients who applied to the polyclinic with the symptoms of DVT (pain and lower extremity swelling) were initially taken into the study. Partial or local obstructions under knee were not taken into study. Diagnosis of DVT was made by a colorDoppler ultrasonography (USG).

A total of 19 patients, with a complete obstruction in the unilateral iliac or main femoral vein and non-massive pulmonary
Correspondence: Mihriban Yalçın, Sahincili Mah. Devlet Hastanesi, 52200 Ordu, Turkey. Tel.: +90.5327838316 - Fax: +90.4522339360.

E-mail: mihribandemir33@hotmail.com

Key words: Balloon angioplasty; deep venous thrombosis; pharmacomechanical thrombectomy.

Acknowledgements: we acknowledge the medical writing assistance provided by American Manuscript Editors (www.americanmanuscripteditors.com) for the final draft of the manuscript.

Contributions: MY, OT, study conception; KE, EGK, data collection; OT, KE, analysis; OT, investigation; MY, EGK, writing. All authors are responsible for critical review and revision, final approval of the article, accountability for all aspects of the work.

Conflict of interest: the authors declare no potential conflict of interest.

Received for publication: 21 April 2018.

Revision received: 24 June 2018.

Accepted for publication: 26 June 2018.

This work is licensed under a Creative Commons Attribution 4.0 License (by-nc 4.0)

(C) Copyright M. Yalcin et al., 2018

Licensee PAGEPress, Italy

Veins and Lymphatics 2018; 7:7513

doi:10.4081/vl.2018.7513

embolism diagnosed with clinical, chest tomography and D-dimer measurements, were treated with PMT, and other fifty-five patients were treated medically (Table 1). All patients had iliofemoral DVT [lower extremity thrombosis (LET) classification class III]. ${ }^{9}$

Patients followed up with medical treatment were treated with low-molecularweight heparin, followed by administration of warfarin with the levels of INR 2-3 after 2 weeks. For patients with insufficient warfarin (treated with warfarin and do not reach recommended PT-INR targets), $20 \mathrm{mg}$ rivaroxaban was administered.

All procedures were performed under local anesthesia. Before the procedure, to avoid a possible complication of pulmonary embolism, an inferior vena cava (IVC) filter (Angel Cathater, BiO2 Medical, San Antonio, TX, USA) was placed via the intact extremity vein, below the renal venous orifices (Figure 1). The patient was then placed prone, an $8 \mathrm{~F}$ sheath was placed in the obstructive vein adjacent the popliteal artery under ultrasound guidance in the popliteal pit, a initial venogram was performed, and 
to maintain the target activating clotting time between 200-250 s unfractionated heparin was administered through the sheath during procedure. In each thrombotic vein segment, the PMT device Cleaner (Rex Medical, Fort Worth, TX, USA) was activated and the thrombotic vein was treated with tissue plasminogen activator (tPA) (Alteplase, Genentech, South San Francisco, CA, USA) injection in $5-10 \mathrm{~cm}$ intervals. After 3 to 5 min of PMT, the device withdrawn. The macerated thrombus material was suctioned. The device was reactivated in segments with partial residual thrombosis. The procedure was repeated up to 3 times for remaining thrombosis for each segment.

Finally a completion venogram was performed (Video 1). On post procedure venography improved target vessel patency, brisk contrast flow and resolved or diminished collateral pathway opacification are the criteria for successful revascularization. ${ }^{10}$ After completion venography all the catheters and wires removed and manual compression is performed for hemostasis.

The technical results were divided into 3 groups: i) complete thrombolysis - more than $90 \%$ of thrombus dissolution; ii) partial thrombolysis - dissolution of thrombus from $50 \%$ to $90 \%$; iii) incomplete thrombolysis less than $50 \%$ thrombus dissolution. ${ }^{11}$

Patients were returned to the clinic at the end of the procedure and were followed up for complications such as bleeding and hematoma. The IVC filters were normally removed, except in five patients. The filter was taken out on the 2 nd day of the procedure in 8 patients, and after 6 months in one patient. In 5 patients who cannot use anticoagulation, due to pre-determined reasons ( 2 patients have cancer, 2 patients have inability to maintain therapeutic anticoagulation and 1 has poor compliance), the filter was left in place. All patients were treated with LMWH and then with warfarin one day after the procedure. Compression therapy was applied to the patients in both groups.

Patients were evaluated clinically at the first week and evaluated with the help of color Doppler USG at 3-month, 6 month and
1 year clinical follow-up. Median follow-up was 16 months (11-20 months). The subjective perception of symptoms was defined by Bozkaya score (complete improvement, partial improvement and no change in clinical status). ${ }^{12}$ Statistical analysis was performed by using SPSS 17.0 (SPSS Inc., Chicago, Illinois).

Continuous data are reported as means and standard deviation. Nominal data are reported as the number of subjects.

\section{Results}

All 19 patients, 14 men and 5 women, presented with pain and lower extremity swelling. The median age was 45 years (range 20-56). Ten patients (53\%) had left and 9 patients $(47 \%)$ had right lower extremity DVT.

After thrombus removal balloon angioplasty was done and underlying stenotic lesions detected. Complete thrombus removal was achieved in 14 patients $(74 \%)$ and the remaining five patients underwent self-expanding stents (Wall stent, Boston Scientific Inc., Galway, Ireland) to achieve full patency (Table 1). Almost all of the patients were treated from a single access site. The common iliac vein was stented in four patients and only the common femoral vein was stented in one patient. An average of $10 \mathrm{mg}$ tPA was given during the procedure.

The pain and swelling complaints of the patients were improved immediately. According to the Bozkaya score, all of the patients had subjective clinical improvement. And there were no cases of major bleeding, acute kidney injury, PE or patient death following the procedure. There was minor subcutaneous hemorrhage at the access side after the procedure in only two patients.

Thirteen patients (68\%) improved without sequel. It was observed with color Doppler USG that full patency was maintained in all patients after three months following PMT.

All patients received at least 3 months of anticoagulation after the procedure. After

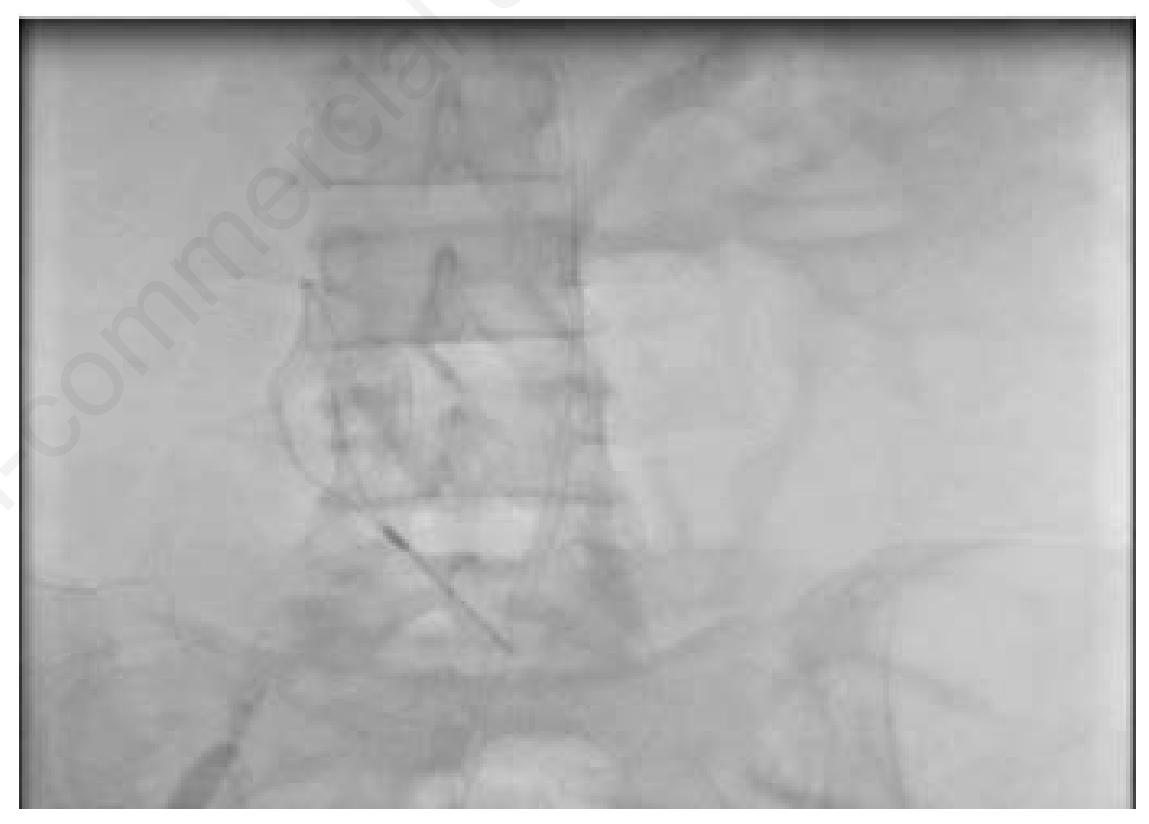

Figure 1. An inferior vena cava filter.

Table 1. Basic characteristics of patients.

\begin{tabular}{lcc} 
& Medical treatment & PMI+Balloon angioplasty and stent \\
Number of patients & 55 & $14+5$ \\
M/F & $39 / 16$ & $14 / 5$ \\
\hline Age (mean) & $48(24-71)$ & $45(20-56)$ \\
Localization of DVT & Right: $24 /$ Left: 31 & Right: $9 /$ Left:10 \\
\hline Initialization of disease & $0-14$ days: $20 />15$ days: 35 & $0-7$ days: $14 / 8-15$ days: $5 />15$ days: No \\
\hline
\end{tabular}

DVT, deep venous thrombosis; F, female; M, male; PMT, pharmacomechanical thrombectomy. 
than five patients (3 patients who were receiving warfarin before the PMT procedure and 2 patients with antithrombin III deficiency) were followed up with Coumadin, one patient was followed up with rivaroxaban (because of poor compliance with anticoagulant medication) while the other 13 patients were followed up with aspirin. In the follow-up period after 6 months and 1 year post procedure, the venous patency rates were $94.7 \%$ (18 patients) and $89.4 \%$ (17 patients) respectively. Because of irregular anticoagulant drug use re-occlusion occurred in 1 patient and was revised with balloon dilation. One patient has died because of myocardial infarction at the end of one year. None of the patients had recurrent DVT.

\section{Discussion}

During the last decade, percutaneous techniques in the aggressive treatment of DVT have been the most promising available methods. They prevent PTS and improve the quality of life. ${ }^{7}$ The use of catheter-directed thrombolysis (CDT) or PMT is recommended by the American College of Chest Physicians (ACCP) in patients with acute onset extensive acute iliofemoral DVT with good functional status, and a life expectancy $\geq 1$ year that have a low risk of bleeding. ${ }^{13}$

There are some series that report improved outcomes by thrombus removal or early thrombus resolution. For example, Cho et $a l .{ }^{14}$ and Rhodes et al. ${ }^{15}$ reported the potential benefits of early thrombus removal.

For acute DVT, PMT has become an effective alternative to open surgical thrombectomies and CDT. ${ }^{16}$ The Cleaner thrombectomy device is one of PMT devices that has a rotating sinusoidal wire or cage for macerating and fragmenting the clot by continuous rotation at approximately 4000 $\mathrm{rpm}$. Reduced drug dose and infusion duration, and so reduced bleeding complications, are further advantages of this method. However, an increased procedure time and a greater risk of venous valvular injury are disadvantages of PMT. ${ }^{17}$

Symptom duration and severity, the anatomic extent of DVT, the presence of signs of circulatory compromise, the patient's bleeding risk profile, life expectancy, and anticipated activity level are the factors for decision on the most appropriate type of treatment. The best candidates are patients with acute iliofemoral DVT. If there is reduced bleeding risk, patients with acute femoropopliteal DVT and significant symptoms may also be good candidates. All of our patients in this study had acute or subacute iliofemoral DVT.

Active bleeding disseminated intravascular coagulation, a recent cerebrovascular event, and neurosurgery or intracranial trauma within three months are absolute contraindications. Recent cardiopulmonary resuscitation, organ biopsy, uncontrolled hypertension, recent major gastrointestinal bleeding within three months, massive pulmonary embolism, and known right-to-left cardiac or pulmonary shunt are relative contraindications. ${ }^{18}$

It was reported in a systematic review that PMT is effective and safe. ${ }^{19}$ Luminal patency achieved in $83-100 \%$ of patients and on mid-term follow-up significant improvements of symptoms achieved in 75$98 \%$ of patients and there were no deaths. Vedantham et al. ${ }^{20}$ reported for iliofemoral DVT that complete lysis was achieved in $31 \%$ of patients. Lee et al. ${ }^{21}$ and Lin et al. ${ }^{22}$ reported $100 \%$ and $70 \%$ complete thrombus removal, respectively. We achieved complete lysis in $74 \%$ of our patients.

Karthikesalingam et al. published a retrospective case series including 481 patients who underwent mechanical thrombectomy. They reported that technical success rate was $82-100 \%$, the incidence of symptomatic pulmonary embolism is $1 \%$ associated with theprocedure and no strokes or deaths were observed. ${ }^{19}$

Bush et al. published the results of 20 patients who underwent pharmacomechanical thrombectomy with the Anjiojet thrombectomy System. They reported $74 \%$ rapid clinical improvement and no complications due to access in any patient. ${ }^{23}$

Chaudhry et al. performed PMT on 28 patients by using the device Trellis system and reported that symptoms were regressed in all patients and venous patency rate was $80 \%$ after the $12^{\text {th }}$ month. ${ }^{24}$

Bozkurt et al. published a study concerning sixteen patients with extensive iliofemoral and/or femoropopliteal acute DVT and managed with the Cleaner device and they reported a $87.5 \%$ success rate. ${ }^{25}$ Koksoy et al. published a study with a success rate (greater than $50 \%$ thrombus resolution) of $97.6 \%$ with the use of the Cleaner device in DVT. ${ }^{26}$

The most common major complication is major bleeding with the incidence of $15 \%$. The other major complications are, intracranial and retroperitoneal bleeding, pulmonary embolism and death. The rheolytic thrombectomy device AngioJet can cause massive intravascular hemolysis, hemoglobinuria, elevated serum potassium level, and acute kidney injury. ${ }^{27}$
In our study there were no major complications, there was minor subcutaneous hemorrhage at the access side after the procedure in only two patients.

Venous angioplasty and/or stenting after PMT may be used under these conditions: flow-limiting iliocaval stenosis; and short segment, residual infrarenal iliocaval thrombosis. ${ }^{9}$ In our study only five patients (26\%) needed balloon angioplasty and additional stenting. We used stents in the iliac position for four patients and in the femoral position for one patient. In our study, the symptoms were also long lasting (greater than 14 days) in patients with stents.

In our present study, 14 patients had complete restoration of antegrade flow (74 $\%$ success rate) and the other five patients underwent balloon angioplasty and additional stenting to achieve significant lysis $(>50 \%)$. The relative lower success rates may be related to the small numbers of patients involved.

\section{Conclusions}

Phlegmasia cerulea dolens, phlegmasia alba dolens, acute/subacute inferior vena cava thrombosis, acute iliofemoral DVT and subacute/chronic iliofemoral DVT are indications for PMT. However, in cases where this treatment is not sufficient, balloon angioplasty and stenting should be used to maintain significant patency.

In conclusion, our single-center experience with short-term results suggests that PMT with the Cleaner device can be used to successfully manage acute and subacute iliofemoral DVT. Randomized trials with long-term follow-up and with a larger numbers of patients are needed for these procedures.

\section{Supplementary material}

Video 1. An example of PMT with Cleaner device.

\section{References}

1. Menon J, Hamilton G. Deep venous thrombosis. Surgery 2007;25:323-6.

2. Kahn SR, Shrier I, Julian JA, et al. Determinants and time course of the post thrombotic syndrome after acute deep venous thrombosis. Ann Intern Med 2008; 149:698-707.

3. Piazza G, Goldhaber SZ. Chronic thromboembolic pulmonary hypertension. N Engl J Med 2011;364: 
351-60.

4. Prandoni P, Lensing AW, Prins MH, et al. Below-knee elastic compression stockings to prevent the post-thrombotic syndrome: A randomized, controlled trial. Ann Intern Med 2004;141:249-56.

5. Delis KT, Bountouroglou D, Mansfield AO. Venous claudication in iliofemoral thrombosis: Long-term effects on venous hemodynamics, clinical status, and quality of life. Ann Surg 2004; 239:118-26.

6. Kahn SR, Ducruet T, Lamping DL, et al. Prospective evaluation of health-related quality of life in patients with deep vein thrombosis. Arch Intern Med 2005; 165:1173-8.

7. Gasparis AP, Labropoulos N, Tassiopoulos AK, et al. Midterm Followup After Pharmacomechanical Thrombolysis for Lower Extremity Deep Venous Thrombosis. Vasc Endovascular Surg 2009;43:61-8.

8. Lookstein R, Giordano C. Deep Vein Thrombosis: Endovascular Management. Mt Sinai J Med J Transl Person Med 2010;77:286-95.

9. Arnoldussen $\mathrm{CW}$, Wittens $\mathrm{CH}$. An imaging approach to deep vein thrombosis and the lower extremity thrombosis classification. Phlebology 2012;27 Suppl 1:143-8.

10. O'Connor P, Lookstein R. Endovascular Interventions for Venous Disease. Tech Vasc Interv Radiol 2018;21:55-64.

11. Gasparis AP, Labropoulos N, Tassiopoulos AK, et al. Midterm followup after pharmacomechanical thrombolysis for lower extremity deep venous thrombosis._Vasc Endovascular Surg 2009;43:61-8.

12. Vasquez MA, Rabe E, McLafferty RB, et al. Revision of the venous clinical severity score: venous outcomes consensus statement: special communication of the American Venous Forum Ad Hoc Outcomes Working Group._J Vasc Surg 2010;52:1387-96.

13. Pappy R, Hanna E, Abu-Fadel M, et al. Isolated Pharmacomechanical Thrombectomy for the Management of Chronic DVT. J Interv Cardiol 2011;24: 99-104.

14. Cho JS, Martelli E, Mozes G, et al. Effects of thrombolysis and venous thrombectomy on valvular competence, thrombogenicity, venous wall morphology, and function. J Vasc Surg 1998;28:787-9.

15. Rhodes JM, Cho JS, Gloviczki P, et al. Thrombolysis for experimental deep venous thrombosis maintains valvular competence and vasoreactivity. J Vasc Surg 2000;31:1193-205.

16. Vedantham S. Interventional approaches to deep vein thrombosis. Am J Hematol 2012;87:S113-8.

17. Malgor RD, Gasparis AP. Pharmacomechanical thrombectomy for early thrombus removal. Phlebology 2012;27:155e62.

18. Vedantham S, Thorpe PE, Cardella JF, et al. Quality improvement guidelines for the treatment of lower extremity deep vein thrombosis with use of endovascular thrombus removal. J Vasc Interv Radiol 2009;20:227-39.

19. Karthikesalingam A, Young EL, Hinchliffe RJ, et al. A systematic review of percutaneous mechanical thrombectomy in the treatment of deep venous thrombosis. Eur J Vasc Endovasc Surg 2011;41:554-65.

20. Vedantham S, Vesely TM, Sicard GA, et al. Pharmacomechanical thrombolysis and early stent placement for iliofemoral deep vein thrombosis. J Vasc Intervent Radiol 2004;15:565-74.

21. Lee KH, Han $\mathrm{H}$, Lee $\mathrm{KJ}$, et al. Mechanical thrombectomy of acute iliofemoral deep vein thrombosis with use of an arrow-trerotola percutaneous thrombectomy device. J Vasc Intervent Radiol 2006;17:487-95.

22. Lin PH, Zhou W, Dardik A, et al. Catheter-direct thrombolysis versus pharmacomechanical thrombectomy for treatment of symptomatic lower extremity deep venous thrombosis. Am J Surg 2006;192:782-8.

23. Bush RL, Lin PH, Bates JT, et al. Pharmacomechanical thrombectomy for treatment of symptomatic lower extremity deep venous thrombosis: safety and feasibility study. J Vasc Surg 2004;40:965-70.

24. Chaudry MA, Pappy R, Hennebry TA. Use of the trellis device in the management of deep vein thrombosis: a retrospective single-center experience. J Invasive Cardiol 2013;25:269-9.

25. Bozkurt A, Kirbas I, Kosehan D, et al. Pharmacomechanical Thrombectomy in the Management of Deep Vein Thrombosis Using the Cleaner Device: An Initial Single-Center Experience. Ann Vasc Surg 2015;29:670-4.

26. Koksoy C, Yilmaz MF, Basbug HS, et al. Pharmacomechanical thrombolysis of symptomatic acute and subacute deep vein thrombosis with a rotational thrombectomy device. J Vasc Interv Radiol 2014;25:1895e900

27. Dukkipati R, Yang EH, Adler S, et al. Acute kidney injury caused by intravascular hemolysis after mechanical thrombectomy. Nat Clin Pract Nephrol 2009;5:112-6. 